\title{
基于近似模型的高速列车头型多目标优化设计 ${ }^{*}$
}

于梦阁 ${ }^{1,2}$ 潘振宽 $^{3}$ 蒋荣超 $^{1}$ 张继业 $^{4}$

(1. 青岛大学机电工程学院 青岛 266071;

2. 青岛大学自动化与电气工程学院系统科学博士后流动站 青岛 266071;

3. 青岛大学计算机科学技术学院 青岛 266071;

4. 西南交通大学汼引动力国家重点实验室 成都 610031)

\begin{abstract}
摘要: 为改善高速列车气动性能, 建立一套高效的多目标气动优化设计方法, 对流线型头型进行多目标气动优化设计。建立 高速列车流线型头型三维参数化模型, 并提取 5 个优化设计变量; 为减少优化设计时间, 利用最优拉丁超立方设计方法在优 化设计空间中进行均匀采样, 利用计算流体力学方法获得对应于各个采样点的气动载荷, 利用 Kriging 代理模型构建优化设 计变量和气动载荷之间的近似模型; 利用多体系统动力学方法计算气动载荷作用下的高速列车轮重减载率; 以气动阻力和轮 重减载率为优化目标, 利用多目标遗传算法 NSGA-II 对高速列车流线型头型进行多目标优化。优化设计变量和优化目标均呈 现收玫的趋势, 采用 Kriging 近似模型优化计算的 Pareto 前沿与采用 CFD(Computational fluid dynamics, CFD)优化计算的 Pareto 前沿较为接近。优化后高速列车的气动阻力最多可降低 $3.27 \%$, 轮重减载率最多可降低 $1.44 \%$, 气动阻力最优的头型与轮重 减载率最优的头型的主要差异在于中部辅助控制线的变化, 前者向内凹, 后者则向外凸。
\end{abstract}

关键词: 高速列车; 气动性能; 多目标优化; Kriging 代理模型; Pareto 前沿

中图分类号: TG156

\section{Multi-objective Optimization Design of the High-speed Train Head Based on the Approximate Model}

\author{
YU Mengge $^{1,2}$ PAN Zhenkuan $^{3}$ JIANG Rongchao ${ }^{1}$ ZHANG Jiye ${ }^{4}$
}

(1. College of Mechanical and Electrical Engineering, Qingdao University, Qingdao 266071;

2. Postdoctoral Research Station of System Science, College of Automation and Electrical Engineering,

Qingdao University, Qingdao 266071;

3. College of Computer Science \& Technology, Qingdao University, Qingdao 266071;

4. State Key Laboratory of Traction Power, Southwest Jiaotong University, Chengdu 610031)

\begin{abstract}
In order to improve the aerodynamic performance of the high-speed train, an efficient multi-objective aerodynamic optimization design method is set up to carry out the multi-objective aerodynamic optimization design of the streamlined head. The three-dimensional parametric model of the streamlined head of the high-speed train is set up, and five optimization design variables are extracted. To reduce the optimization time, the optimal Latin hypercube design method is used for the uniform sampling in the optimization design space, and the aerodynamic loads corresponding to each sampling point are obtained through the computational fluid dynamic method. The Kriging surrogate model is used to construct the approximate model between optimization design variables and aerodynamic loads. The load reduction factor of the high-speed train caused by the aerodynamic loads is computed by the multi-body system dynamic method. Then the aerodynamic drag force and load reduction factor are set as optimization objectives and the multi-objective optimization of the high-speed train head is conducted by the multi-objective genetic algorithm NSGA-II. The optimization design variables and optimization objectives show the tendency of convergence. The Pareto frontier computed by the Kriging approximate model is close to that computed by the computational fluid dynamics (CFD). After optimization, the aerodynamic drag of the optimized train is reduced by up to $3.27 \%$, and the load reduction factor is reduced
\end{abstract}

* 国家自然科学基金(51705267, 51605397)、中国博士后科学基金 (2018M630750)和山东省自然科学基金(ZR2014EEP002)资助项目。 20180908 收到初稿, 20190605 收到修改稿 
by up to $1.44 \%$. As for the optimal head with minimum aerodynamic drag force and the optimal head with minimum load reduction factor, the main difference is the deformation of the central auxiliary control line, with the former concave and the latter convex.

Key words: high-speed train; aerodynamic performance; multi-objective optimization; Kriging surrogate model; Pareto frontier

\section{0 前言}

随着列车运行速度的不断提高, 高速列车的动 态环境发生质的改变, 由机械、电气作用为主, 变 成以气动作用为主, 解决空气动力学问题已经成为 高速列车设计研发的关键问题 ${ }^{[1-4]}$ 。列车气动阻力与 运行速度的平方成正比, 当列车运行速度达到 $250 \sim 300 \mathrm{~km} / \mathrm{h}$, 气动阻力在总阻力中所占的比例将 超过 $75 \%$, 气动阻力已经成为制约列车提速和节能 的重要因素之一。减小气动阻力是高速列车头型设 计的重要考虑因素。然而, 气动阻力的减小可能会 引起其他气动力和气动力矩增大, 进而可能影响到 高速列车的运行安全特性。因此, 减小列车气动阻 力, 并提高列车的运行安全特性是高速列车流线型 头型优化设计的关键问题。

传统的高速列车头型设计思路通常是优选法, 即先拟定出各种头型方案, 再通过风洞试验、动 模型试验、数值计算等方法进行比较选择及设计 完善 ${ }^{[5-6]}$ 。优选法过多的依赖于工程经验, 且最终选 定的头型也并不一定是最优的。近年来, 直接优化 方法得到国内外学者的青睐。直接优化设计是在满 足一定约束条件的前提下, 采用数学方法对某些设 计目标寻求尽可能最大或最小(如气动阻力最小化、 气动升力最小化等)。KWON 等 ${ }^{[7]}$ 研究了鼻部形状对 隧道压力波的影响, 并基于响应面法对高速列车的 鼻部形状进行优化设计, 分析结果表明通过对鼻部 的优化设计可以较好地降低隧道压力波强度。

MUÑOZ-PANIAGUA 等 ${ }^{[8]}$ 结合遗传算法和径向基神 经网络, 以降低高速列车过隧道时的最大压力梯度 和气动阻力为目标, 对高速列车的鼻部形状分别进 行了单目标和多目标优化。KRAJNOVIC 等 ${ }^{[9]}$ 提出 采用响应面法对列车气动特性进行优化, 并分别针 对横风稳定性和气动阻力, 研究三种响应面模型: 多项式函数、径向基神经网络、径向基神经网络和 多项式函数的组合模型, 研究表明组合模型可以取 得较好的优化效果。KU 等 ${ }^{[10]}$ 以微气压波为优化目 标, 获取头车流线型部分的最优截面变化率, 然后 保证截面变化率不变, 对头车气动阻力进行优化设 计。YAO 等 ${ }^{[11]}$ 构建了包括 33 个优化设计变量的高 速列车头型参数化模型, 并结合支持向量机回归模
型和多目标粒子群算法对高速列车头型进行优化设 计, 优化后列车气动阻力降低 $13.62 \%$, 尾车升力 降低 $9.84 \%$ 。熊骏等 ${ }^{[12]}$ 以侧风下头车的倾覆力矩和 列车明线气动阻力为优化目标, 采用遗传算法和近 似模型相结合的方法, 对城际动车组的外形进行气 动优化设计。张亮等 ${ }^{[13]}$ 以整车气动阻力和头车最大 表面声功率为优化目标, 对流线型头型进行多目标 自动气动优化设计。LI 等 ${ }^{[14]}$ 采用自由变形技术对 高速列车头型进行参数化设计, 以气动阻力和气动 升力为优化目标, 对高速列车头型进行多目标优化 设计。

以上研究工作均针对部分气动性能对高速列车 头型进行优化, 但某些气动力(矩)的减小可能会引 起其他气动力(矩)增加, 如气动阻力的减小极有可 能引起气动升力和点头力矩的恶化, 并最终影响到 列车的运行安全性。因此, 在高速列车减阻优化时, 同时将气动阻力和运行安全指标作为优化目标进 行高速列车头型优化设计可以给出更好的优化结 果。此外, 在高速列车流线型头型的优化设计过程 中, 每一个优化迭代步内, 都需要进行一次高速列 车空气动力学计算, 而空气动力学计算非常耗时, 从而使得整个高速列车流线型头型优化设计周期 非常长。因此, 构建精确高效的高速列车空气动力 学近似计算模型也是非常必要的。基于此, 本文结 合计算流体力学、最优拉丁超立方设计、Kriging 代理模型、车辆系统动力学和多目标优化算法, 建 立一套高效精确的高速列车流线型头型多目标优 化设计方法, 以气动阻力和轮重减载率为优化设计 目标, 对高速列车流线型头型进行多目标自动优化 设计。

\section{1 优化设计流程}

由于高速列车空气动力学计算非常耗时, 为减 少高速列车流线型头型优化设计时间, 本文拟利用 Kriging 代理模型构造满足工程计算精度要求的高 速列车空气动力学近似计算模型, 在每一个迭代步 内, 当优化设计变量的值更新后, 可以利用 Kriging 代理模型计算气动载荷的数值, 减少网格划分时间 和空气动力学计算时间, 从而可以极大地缩短高速 列车流线型头型优化设计周期。 
本文高速列车流线型头型优化设计流程主要分 为两步, 如图 1 所示。第一步建立高速列车空气动 力学计算的 Kriging 代理模型, 具体而言, 利用试 验设计方法在优化设计变量的设计空间中均匀采 样, 根据采样点进行高速列车流线型头型的三维参 数化几何建模, 获得参数化模型文件; 根据参数化 模型文件, 进行计算网格划分, 得到网格文件; 利 用网格文件, 进行高速列车空气动力学计算, 得到 气动载荷; 利用 Kriging 代理模型建立优化设计变 量和气动载荷之间的近似计算模型。第二步基于 Kriging 代理模型、车辆系统动力学和多目标遗传算 法进行多目标气动优化设计。

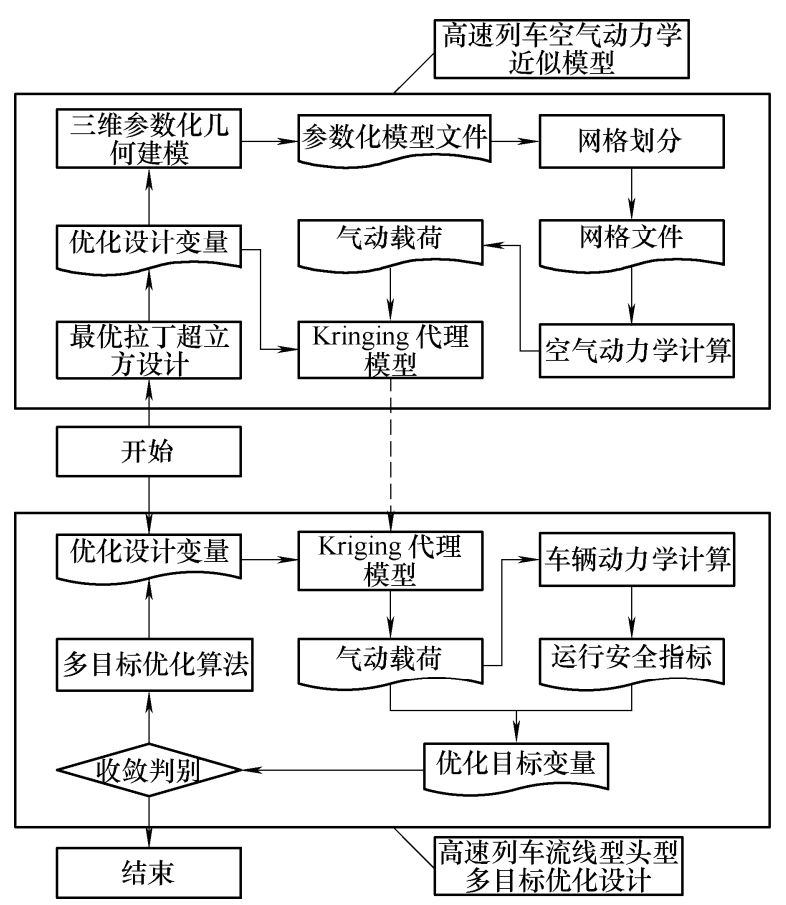

图 1 基于近似模型的多目标气动优化流程

\section{2 高速列车三维参数化模型}

由于高速列车流线型头型具有高度的对称性, 因此本文对列车左半部流线型头型进行参数化建 模。根据某高速列车流线型头部外形, 利用头型表 面控制点, 建立 12 条样条曲线(编号为 $\mathrm{C} 1 \sim \mathrm{C} 12$ ), 利用样条曲线生成 7 个样条曲面，进而构成流线型 头型外形 ${ }^{[2]}$, 如图 2 所示。本文选取 5 个流线型头 型优化设计变量, 即 $d z_{1} 、 d y_{3} 、 d y_{4} 、 d y_{7}$ 和 $n_{s}$, 分别 对应于纵向对称线 $\mathrm{C} 1$ 、水平最大外轮廓线 C3、车 底水平轮廓线 C4、中部辅助控制线 C7 和鼻尖高度 控制线 C9。根据工程经验, 各变量的取值范围及含 义如表 1 所示, 各曲线的具体变形方式与文献[2]相 一致。

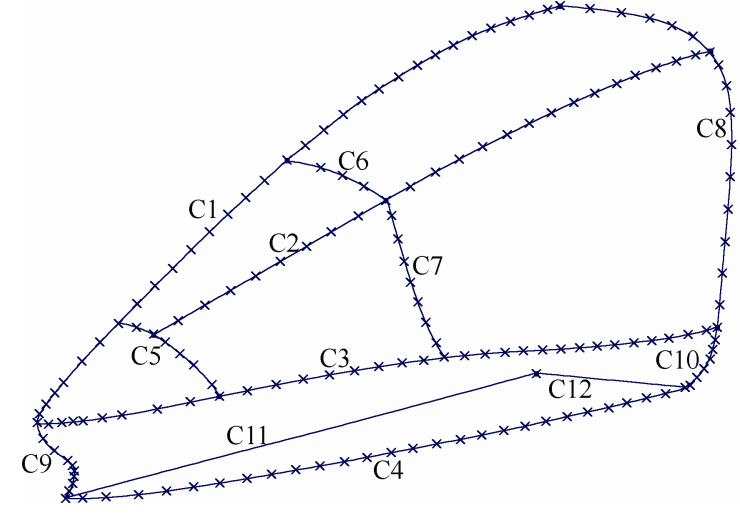

图 2 左半部流线型头型模型

表 1 流线型头型设计变量的取值范围及意义

\begin{tabular}{|c|c|c|}
\hline 变量 & 取值范围 & 变量含义 \\
\hline$d z_{1}$ & {$[-400,400] \mathrm{mm}$} & $\begin{array}{l}d z_{1}>0 \text { 表示 } \mathrm{C} 1 \text { 纵向中点向上移动 } \\
d z_{1}<0 \text { 表示 } \mathrm{C} 1 \text { 纵向中点向下移动 }\end{array}$ \\
\hline$d y_{3}$ & {$[-100,200] \mathrm{mm}$} & $\begin{array}{l}d y_{3}>0 \text { 表示 C3 纵向中点向纵向对称面移动 } \\
d y_{3}<0 \text { 表示 C3 纵向中点远离纵向对称面移动 }\end{array}$ \\
\hline$d y_{4}$ & {$[-200,200] \mathrm{mm}$} & $\begin{array}{l}d y_{4}>0 \text { 表示 C4 纵向中点向纵向对称面移动 } \\
d y_{4}<0 \text { 表示 C4 纵向中点远离纵向对称面移动 }\end{array}$ \\
\hline$d y_{7}$ & {$[-0.2,0.4]$} & $\begin{array}{l}d y_{7}>0 \text { 表示 C7 向外凸 } \\
d y_{7}<0 \text { 表示 C7 向内凹 }\end{array}$ \\
\hline$n_{s}$ & {$[0.8,1.2]$} & $\begin{array}{l}n_{s}>1 \text { 表示鼻尖变高 } \\
n_{s}<1 \text { 表示鼻尖变低 }\end{array}$ \\
\hline
\end{tabular}

\section{3 高速列车空气动力学模型}

\section{1 数值计算模型}

高速列车绕流流场计算采用不可压缩定常流， 湍流模型采用标准 $k-\varepsilon$ 湍流模型，控制方程为

$$
\operatorname{div}(\rho \boldsymbol{u} \boldsymbol{\varphi})=\operatorname{div}(\Gamma \operatorname{grad}(\boldsymbol{\varphi}))+\boldsymbol{S}
$$

式中, $\operatorname{div}(\cdot)$ 表示散度; $\operatorname{grad}(\cdot)$ 表示梯度; $\rho$ 表示 密度; $\boldsymbol{u}$ 表示速度矢量; $\boldsymbol{\varphi}$ 表示流场通量； $\boldsymbol{S}$ 表示 源项; $\Gamma$ 表示扩散系数。

高速列车模型采用 “头车 + 中间车 + 尾车” 编 组, 流场计算区域如图 3 所示, 列车前端鼻雉与计 算区域入口之间的距离为 $100 \mathrm{~m}$, 列车纵向对称面

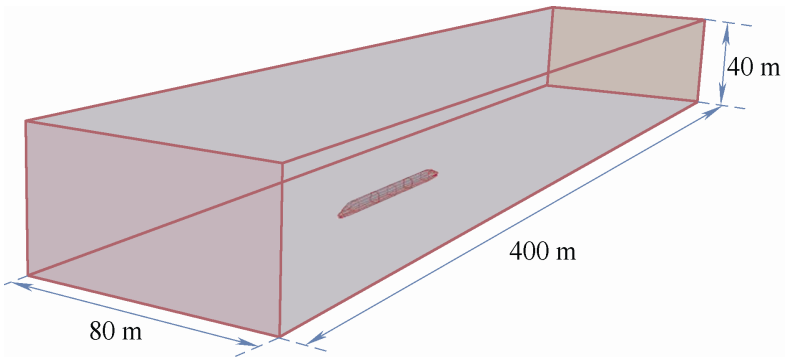

图 3 计算区域 
与计算区域两侧之间的距离为 $40 \mathrm{~m}$, 列车底部与地 面之间的距离为 $0.376 \mathrm{~m}$ 。计算区域左侧设置为速 度入口, 右侧设置为压力出口, 两侧及顶端设置为 对称边界, 底端设置为滑移壁面, 滑移速度与入口 速度相同, 以模拟地面效应。列车表面设置为静止 壁面。

\section{2 计算方法验证}

为验证数值计算的准确性, 将数值计算结果与 风洞试验结果进行对比。风洞试验模型为 $1: 8$ 三车 编组的缩比列车模型, 模型中包括转向架和风挡, 并考虑了轨道和路基。数值计算的几何模型与风洞 试验模型一致, 数值计算区域与风洞尺寸一致。图 4 给出气动阻力的数值计算结果与风洞试验结果的 对比 ${ }^{[15]}$ 。由图 4 可以看出, 各节车气动阻力系数的 计算值与试验值吻合较好, 表明本文的计算方法能 够较好地模拟列车的流动特性。

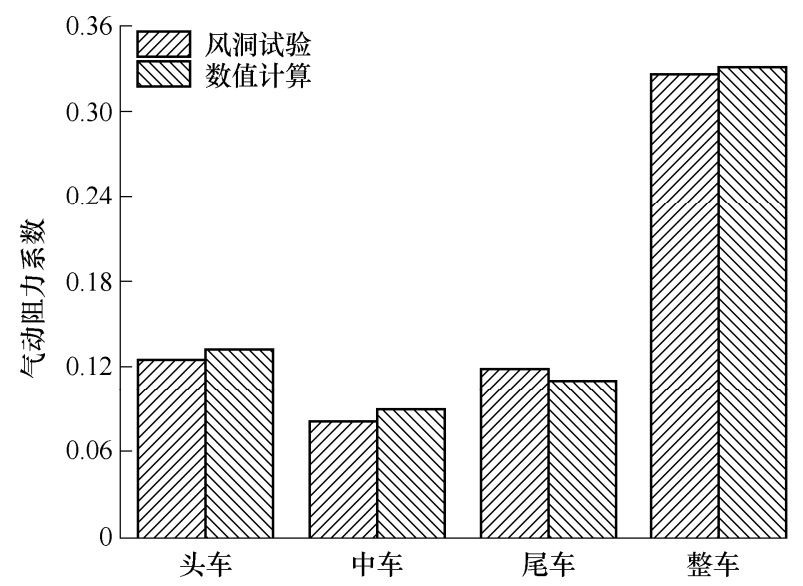

图 4 计算值与试验值的对比

\section{3 网格无关性验证}

计算网格应具有独立性, 即加密计算网格时, 流场计算结果变化不大。为此, 针对 3.1 节建立的 数值计算模型, 在列车表面设置不同尺寸的网格, 研究网格尺寸对计算结果的影响。本文共划分三套 计算网格: 三套网格的列车表面的面网格最大尺寸 分别为 $250 \mathrm{~mm} 、 200 \mathrm{~mm} 、 150 \mathrm{~mm}$, 列车表面采用 三角形网格; 计算空间采用四面体网格, 体网格最 大尺寸为 $4000 \mathrm{~mm}$ 。表 2 给出三套网格下的列车气 动阻力和气动升力。由表 2 可以看出, 当面网格 尺寸由 $250 \mathrm{~mm}$ 加密到 $200 \mathrm{~mm}$ 时, 气动阻力减 小 $4.55 \%$, 气动升力减小 $6.22 \%$, 当面网格尺寸由

表 2 不同网格尺寸的计算结果

\begin{tabular}{cccc}
\hline & \multicolumn{3}{c}{ 表面网格尺寸 $/ \mathrm{mm}$} \\
\cline { 2 - 4 } & 250 & 200 & 150 \\
\hline 气动阻力/ $\mathrm{N}$ & 3456 & 3299 & 3327 \\
气动升力/ $\mathrm{N}$ & -3465 & -3250 & -3219 \\
\hline
\end{tabular}

$200 \mathrm{~mm}$ 加密到 $150 \mathrm{~mm}$ 时, 气动阻力增加 $0.86 \%$, 气动升力减小 $0.94 \%$ 。因此, 当列车表面的面网格 最大尺寸为 $200 \mathrm{~mm}$ 时, 继续加密计算网格对流场 计算结果的影响已非常小。因此, 本文采用第二套 网格进行高速列车空气动力学计算。

\section{4 列车空气动力学近似模型}

\section{1 最优拉丁超立方设计}

为使高速列车空气动力学近似模型能够较好 地估算设计空间中任意点的气动载荷, 需采用一 组较好的输入变量, 这组输入变量应尽可能均匀 分布在设计空间中。为此, 本文采用最优拉丁超 立方设计方法, 此方法可使得所有试验点尽量均 匀分布在设计空间中，具有很好的空间填充性和 均衡性 ${ }^{[16]}$ 。

图 5 以头型设计变量 $d z_{1}$ 为例, 给出优化设计 变量的试验设计点。由图 5 可以看出, $d z_{1}$ 在其设计 空间中的分布较为均匀。图 6 给出与试验设计点相 对应的高速列车头车气动阻力及气动升力。

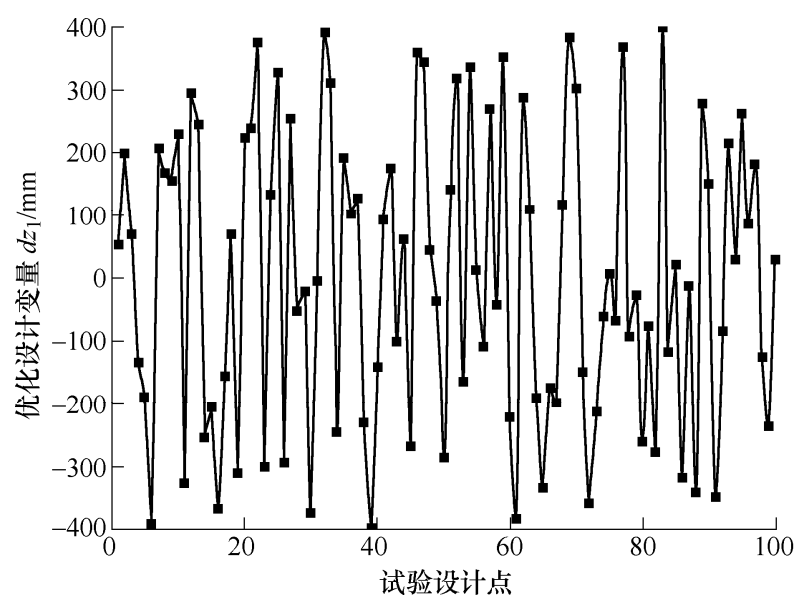

图 5 优化设计变量 $d z_{1}$ 的试验设计点

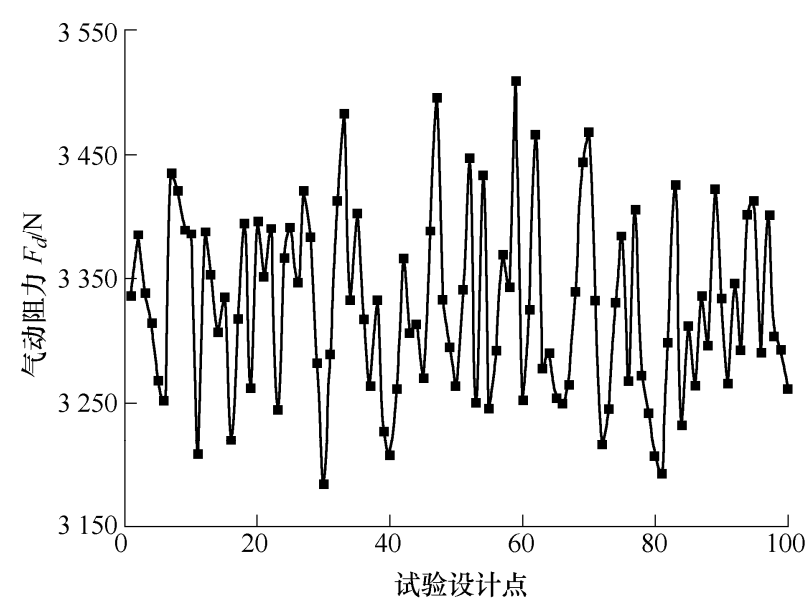

(a) 气动阻力 


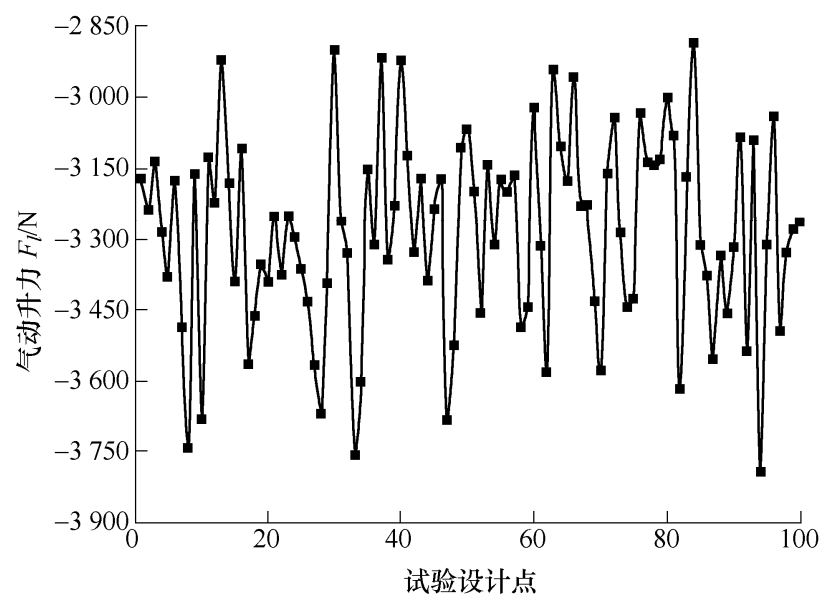

(b) 气动升力

图 6 各个试验设计点下的气动力

图 7 给出高速列车头车的气动阻力和气动升力 与头型设计变量的相关系数。相关系数是研究变量 之间线性相关程度的量, 其取值范围为 $[-1,1]$, 相关 系数为正值表示变量之间具有正相关关系, 相关系 数为负值表示变量之间具有负相关关系, 且其绝对 值越大表示相关程度越大。

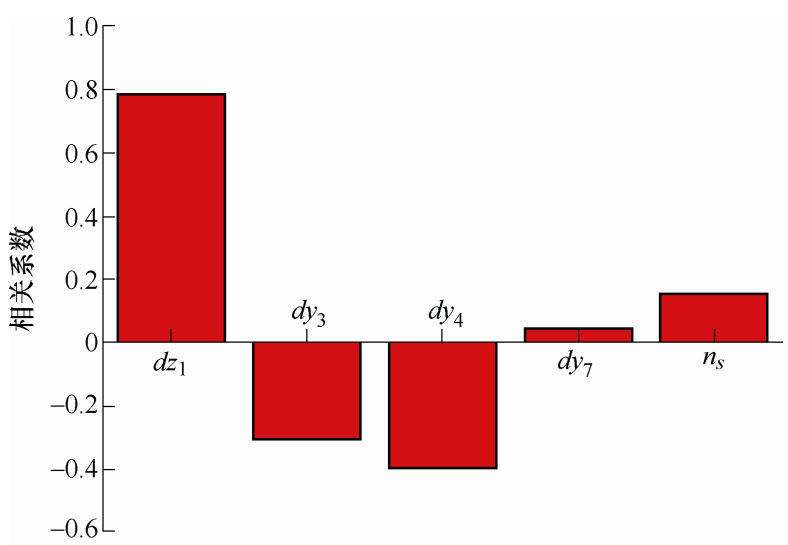

(a) 气动阻力与优化设计变量的相关性

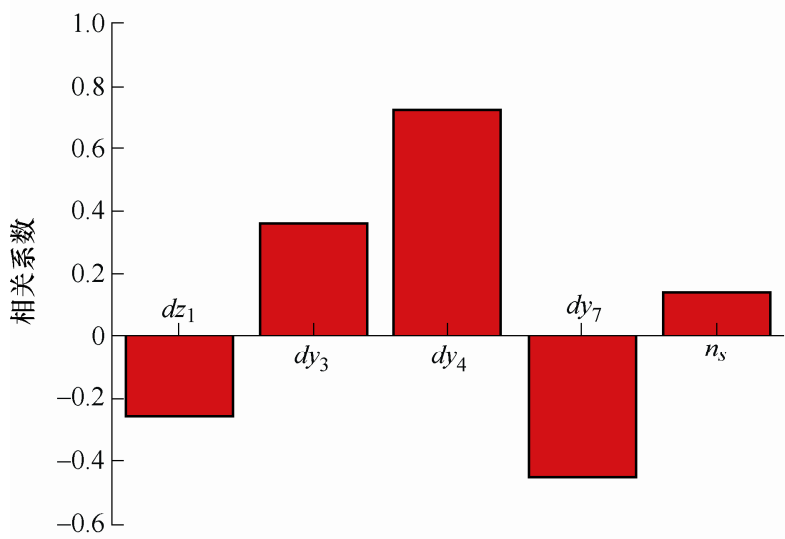

(b) 气动升力与优化设计变量的相关性

图 7 优化目标和优化设计变量的相关性

由图 7 可以看出, 头型设计变量 $d z_{1} 、 d y_{3} 、 d y_{4}$ 、 $d y_{7}$ 对气动阻力和气动升力的影响是相反的, 而 $n_{s}$
对气动阻力和气动升力的影响是相同的。通过分析 可以发现，气动阻力和气动升力具有负相关性，相 关系数为 -0.6202 。因此, 头型设计变量在改善气动 阻力的同时极有可能导致气动升力的恶化, 属于典型 的多目标优化设计问题。气动阻力与 $d z_{1} 、 d y_{7}$ 和 $n_{s}$ 在 一定范围内具有正相关性，与 $d y_{3}$ 和 $d y_{4}$ 在一定范围 内具有负相关性, 即当 C1 纵向中点向下移动, C7 向内凹, 鼻尖高度变小, C3 和 C4 纵向中点向纵向 对称面移动时, 气动阻力呈减小趋势。气动升力与 $d z_{1}$ 和 $d y_{7}$ 在一定范围内具有负相关性, 与 $n_{s} 、 d y_{3}$ 和 $d y_{4}$ 在一定范围内具有正相关性, 即当 C1 纵向中点 向上移动, C7 向外凸, 鼻尖高度变小, C3 和 C4 纵向 中点远离纵向对称面移动时, 气动升力呈减小趋势。

\subsection{Kriging 代理模型构造}

Kriging 代理模型是从变量相关性和变异性出 发, 在有限区域内对目标变量的取值进行无偏、最 优估计的一种方法。Kriging 代理模型由全局模型和 局部偏差结合而成 ${ }^{[17]}$, 可以表示为

$$
y(\boldsymbol{x})=f(\boldsymbol{x})+z(\boldsymbol{x})
$$

式中, $\boldsymbol{x}$ 表示设计变量; $y(\boldsymbol{x})$ 表示目标函数; $f(\boldsymbol{x})$ 表示确定性函数, 由于随机部分已经具有足够的能 力捕捉目标函数的变化趋势, 因此本文将 $f(\boldsymbol{x})$ 取 为常数 $\mu ; z(x)$ 表示随机分布的误差, 提供对模拟 局部偏差的近似, 其均值为 0 , 方差为 $\sigma_{z}^{2}$, 协方差 矩阵为

$$
\operatorname{Cov}\left[z\left(\boldsymbol{x}^{i}\right), z\left(\boldsymbol{x}^{j}\right)\right]=\sigma_{z}^{2}\left[\boldsymbol{R}\left(\boldsymbol{\theta}, \boldsymbol{x}^{i}, \boldsymbol{x}^{j}\right)\right]
$$

式中, $\operatorname{Cov}(\cdot, \cdot)$ 表示协方差; $R\left(\boldsymbol{\theta}, \boldsymbol{x}^{i}, \boldsymbol{x}^{j}\right)$ 表示带有 参数 $\boldsymbol{\theta}$ 的两个样本点 $\boldsymbol{x}^{i}$ 和 $\boldsymbol{x}^{j}$ 之间的相关函数; $i$, $j$ 表示序号, $i, j=1,2, \cdots, l ; l$ 表示样本点的个数; $\boldsymbol{x}^{i}$ 表示第 $i$ 个样本点; $\boldsymbol{x}^{j}$ 表示第 $j$ 个样本点。相关 函数的类型有很多, 本文采用最常用的 Gauss 函数 作为相关函数, 其表达式为

$$
\boldsymbol{R}\left(\boldsymbol{\theta}, \boldsymbol{x}^{i}, \boldsymbol{x}^{j}\right)=\exp \left[-d\left(\boldsymbol{x}^{i}, \boldsymbol{x}^{j}\right)\right]
$$

式中, $d\left(\boldsymbol{x}^{i}, \boldsymbol{x}^{j}\right)$ 表示样本点 $\boldsymbol{x}^{i}$ 和 $\boldsymbol{x}^{j}$ 的距离。

$$
d\left(\boldsymbol{x}^{i}, \boldsymbol{x}^{j}\right)=\sum_{k=1}^{m} \theta_{k}\left|x_{k}^{i}-x_{k}^{j}\right| \quad i, j=1,2, \cdots, m
$$

式中, $m$ 表示设计变量个数; $x_{k}^{i}$ 表示第 $i$ 个样本点 $\boldsymbol{x}^{i}$ 的第 $k$ 个设计变量的值; $x_{k}^{j}$ 表示第 $j$ 个样本点 $\boldsymbol{x}^{j}$ 的第 $k$ 个设计变量的值; $\theta_{k}$ 表示未知的相关参数的 权因子。

为提高预测精度及模型的泛化能力, Kriging 模 型要求预测误差的均值等于 0 , 预测误差的标准差 最小, 由此得到如下函数 


$$
-\frac{1}{2} n \ln \left(\hat{\sigma}_{z}^{2}\right)-\frac{1}{2} \ln (|\boldsymbol{R}|)
$$

通过优化方法找到最优的 $\theta_{k}$ 值, 使得此函数最 大化, 由此构造的 Kriging 代理模型精度最高。本 文采用遗传算法求解式(6)的最大化问题, 以获得最 优的 $\theta_{k}$ 值。

高速列车明线运行气动载荷主要是气动阻力、 气动升力和点头力矩, 因此需构建 3 个 Kriging 代 理模型，这 3 个模型的最优的 $\theta_{k}$ 值如表 3 所示。 $\theta_{k}$ 的大小反映了设计变量对目标函数的影响程度, 可 衡量设计变量对目标函数的重要性。由表 3 可以看 出, 各个设计变量对应的 $\theta_{k}$ 值有较大不同。对于气 动阻力, 设计变量的重要性依次为 $d z_{1} 、 d y_{4} 、 d y_{3}$ 、 $n_{s} 、 d y_{7}$ 。对于气动升力, 设计变量的重要性依次为 $d y_{4} 、 d y_{7} 、 d y_{3} 、 n_{s} 、 d z_{1}$ 。对于点头力矩, 设计变量 的重要性依次为 $d z_{1} 、 d y_{7} 、 d y_{3} 、 d y_{4} 、 n_{s}$ 。由此可知, 设计变量对不同目标函数的影响程度并不一致, 比 如 $d z_{1}$ 对气动阻力和点头力矩的影响程度最大, 而 对气动升力的影响程度最小。

表 3 Kriging 代理模型的最优 $\theta_{\boldsymbol{k}}$ 值

\begin{tabular}{lccccc}
\hline & $\theta_{1}$ & $\theta_{2}$ & $\theta_{3}$ & $\theta_{4}$ & $\theta_{5}$ \\
\hline 设计变量 & $d z_{1}$ & $d y_{3}$ & $d y_{4}$ & $d y_{7}$ & $n_{\mathrm{s}}$ \\
气动阻力 $/ \mathrm{N}$ & 1.4644 & 0.3288 & 0.3561 & 0.1292 & 0.2485 \\
气动升力 $/ \mathrm{N}$ & 0.2762 & 0.3191 & 0.7968 & 0.3816 & 0.2867 \\
点头力矩 $/(\mathrm{N} \cdot \mathrm{m})$ & 0.7443 & 0.2948 & 0.2850 & 0.3053 & 0.2331 \\
\hline
\end{tabular}

图 8 给出了气动阻力、气动升力和点头力矩的 Kriging 计算值与 CFD(Computational fluid dynamics, CFD)计算值之间的比较, 图中直线为 $45^{\circ}$ 对角线, 代表 Kriging 计算值与 CFD 计算值相等。由图 8 可 以看出, 气动阻力、气动升力和点头力矩的 Kriging 计算值与 CFD 计算值的差异较小, 误差均小于 $2 \%$ 。 基于 Kriging 代理模型构建的高速列车空气动力学 近似模型具有很好的预测精度, 可以满足工程计算 精度要求。

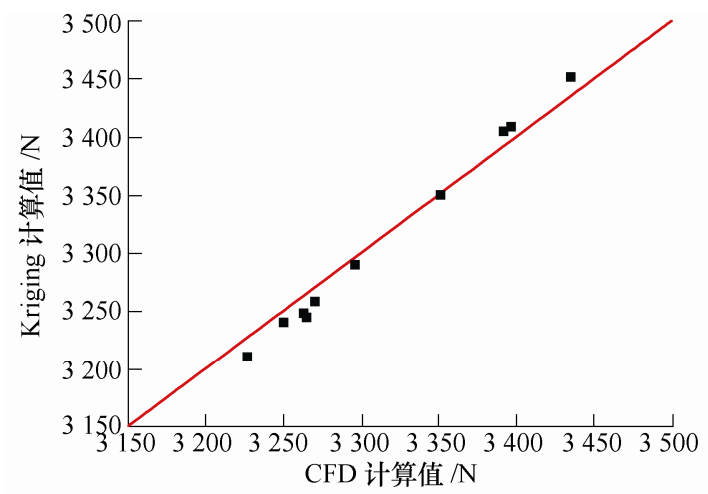

(a) 气动阻力

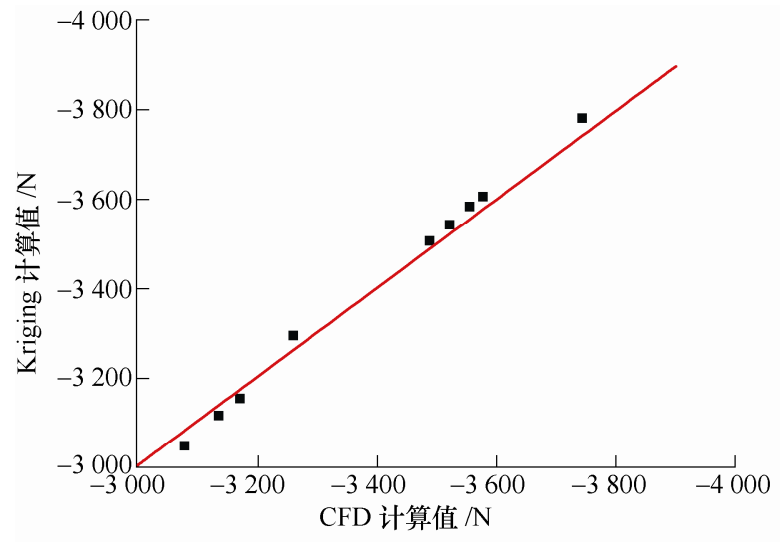

(b) 气动升力

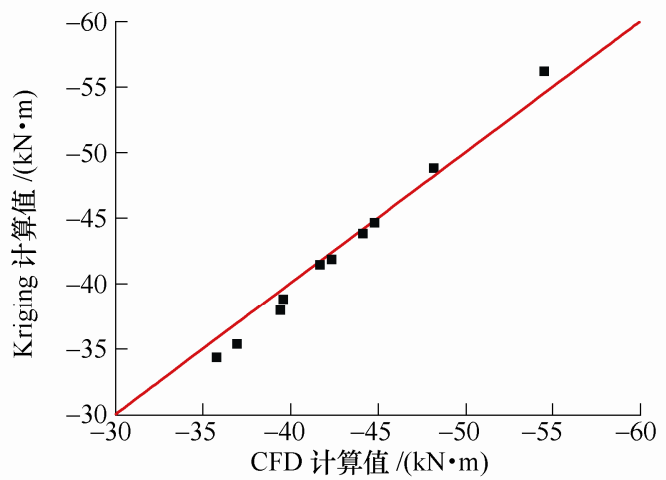

(c) 点头力矩

图 8 Kriging 计算值与 CFD 计算值的比较

\section{5 车辆系统动力学模型}

参照国内某高速列车动力学参数, 利用多体系 统动力学软件 SIMPACK 建立高速列车多体系统动 力学模型 ${ }^{[18]}$ 。轨道车辆的多体系统动力学主要包括 车辆动力学和轮轨关系两部分。单节车辆模型由 1 个车体, 2 个构架, 4 个轮对和 8 个转臂组成, 整个 车辆动力学模型共有 50 个自由度。轮轨关系包括轮 轨几何关系和轮轨接触力两部分。模型中高速列车 的车轮踏面采用 LMA 踏面, 钢轨采用 T60 钢轨。 轮轨接触力的计算采用 Kaller 理论。模型中的轨道 不平顺采用国内某高速铁路实测轨道谱。

将气动升力和点头力矩作为外加载荷作用于高 速列车系统动力学模型中, 以分析高速列车的运行 安全性。根据高速列车整车运行规范, 高速列车的 运行安全评价指标主要包括脱轨系数、轮重减载率、 轮轴横向力和轮轨垂向力。车辆系统动力学计算时, 将轨道考虑为直线轨道, 并选取四个轮对的轮重减 载率的最大值作为优化目标。

\section{6 数值计算结果分析}

采用多目标遗传算法 NSGA-II 求解高速列车流 
线型头型多目标优化设计问题 ${ }^{[19]}$ 。高速列车运行速 度为 $300 \mathrm{~km} / \mathrm{h}$, 流线型头型优化设计变量见表 1 , 优化目标为头车气动阻力和轮重减载率, 均为最小 化优化。多目标优化计算时, NSGA-II 算法的初始 采样点设定为 12 个, 并进行 25 代遗传计算, 从而 在完成 300 个设计后, 高速列车流线型头型优化设 计计算完毕。

图 9 给出优化过程中优化设计变量 $d z_{1}$ 的整体 变化曲线, 由图 9 可以看出, 通过优化算法在设计 空间中的采样, 优化设计变量呈现收玫的趋势。通 过反复的迭代计算, 多目标遗传算法可以寻找到使 得目标值最小化的 Pareto 最优设计点。

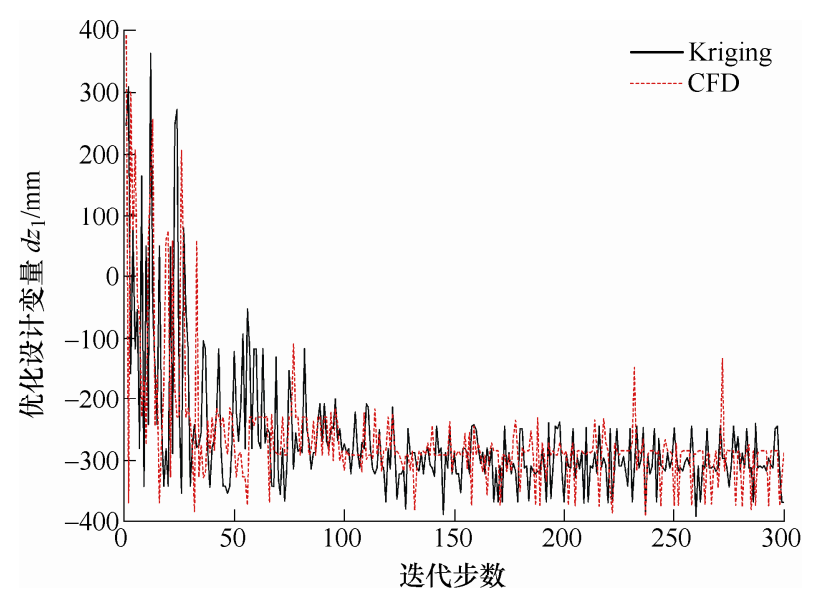

图 9 优化设计变量 $d z_{1}$ 的历史曲线

图 10 以气动阻力为例, 给出优化过程中优化目 标的整体变化曲线。由图 10 可以看出, 通过优化 算法在设计空间中的采样, 气动阻力整体呈减小趋 势, 高速列车流线型头型逐渐向气动阻力最优的方 向改进。

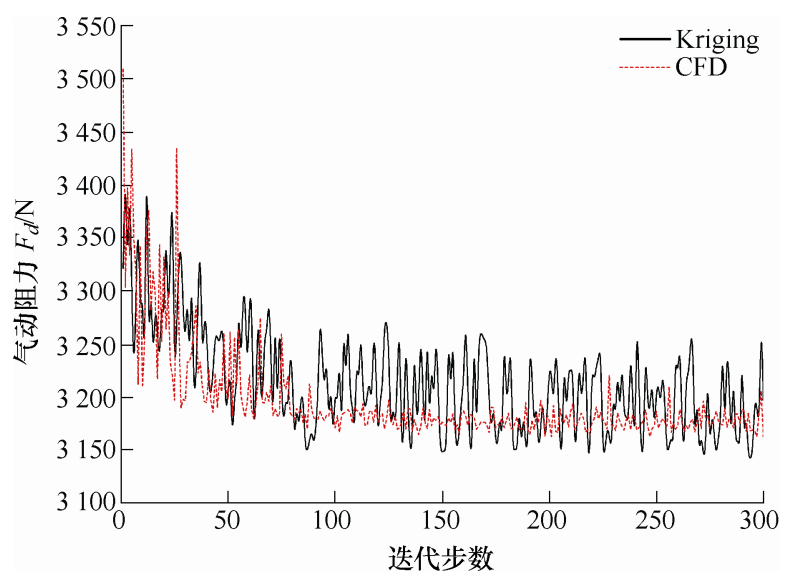

图 10 气动阻力的历史曲线

图 11 给出高速列车流线型头型多目标优化设 计的 Pareto 前沿, 图中五角星“丸”表示与初始流线 型头型相对应的气动阻力和轮重减载率。由图 11 可知, 经过对流线型头型进行多目标优化设计, 高
速列车的气动阻力和轮重减载率均得到改善, 多目 标优化取得较好的效果。通过 Kriging 近似模型计 算得到的 Pareto 前沿与通过 CFD 方法计算得到的 Pareto 前沿较为接近, 近似模型能够获得较好的优 化结果。

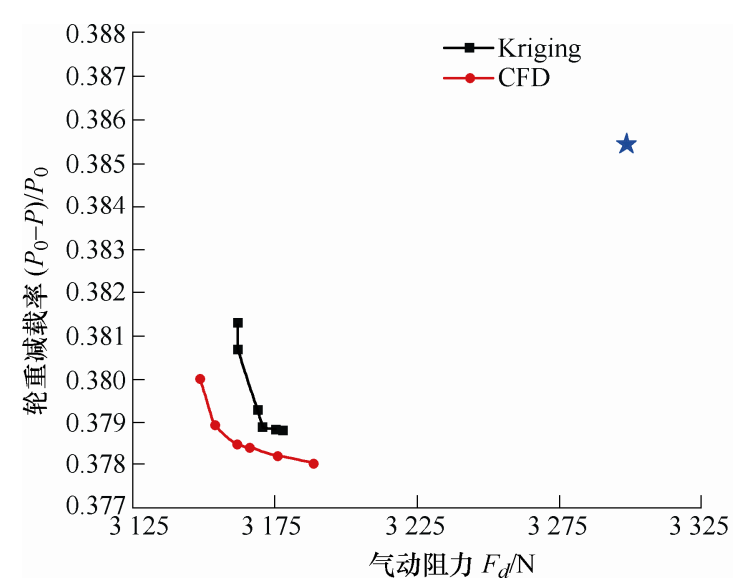

图 11 Pareto 前沿

表 4 给出 Pareto 前沿上的气动阻力和轮重减载 率的 Kriging 计算值与 CFD 计算值的比较。由表 4 可知, Kriging 计算值与 CFD 计算值较为接近, 误 差小于 $2 \%$ 。优化后, 气动阻力较原始值最多可降 低 $3.27 \%$, 轮重减载率较原始值最多可降低 $1.44 \%$ 。

表 4 Pareto 前沿上近似值与实际值的对比

\begin{tabular}{cccccccc}
\hline \multirow{2}{*}{$\begin{array}{c}\text { Pareto } \\
\text { 最优解 }\end{array}$} & \multicolumn{3}{c}{ 气动阻力 } & & \multicolumn{3}{c}{ 轮重减载率 } \\
\cline { 2 - 4 } \cline { 7 - 8 } Priging & CFD & 误差(\%) & Kriging & CFD & 误差 \\
\hline P2 & 3149 & 3191 & -1.32 & & 0.3800 & 0.3838 & $-0.99 \%$ \\
P3 & 3154 & 3204 & -1.56 & & 0.3786 & 0.3832 & $-1.12 \%$ \\
P4 & 3162 & 3201 & -1.22 & & 0.3785 & 0.3825 & $-1.06 \%$ \\
P5 & 3166 & 3202 & -1.12 & & 0.3784 & 0.3830 & $-1.20 \%$ \\
P6 & 3176 & 3213 & -1.15 & 0.3782 & 0.3821 & $-1.02 \%$ \\
\hline
\end{tabular}

图 12 和图 13 分别给出气动阻力最小的头型 (P1)、轮重减载率最小的头型(P6)与初始头型的对 比。由图 12 和图 13 可以看出, 与初始头型相比, 两个最优头型的纵向对称线 C1 纵向中点均向下移 动, 车底水平外轮廓线 $\mathrm{C} 4$ 纵向中点均向纵向对称 面移动, 且两个最优头型的 C1 和 C4 的变化值相差 不大。两个最优头型的主要差异在于中部辅助控制 线 C7 和水平最大外轮廓线 C3 的变化。对于中部辅 助控制线 C7, 最优头型 P1 相对于初始头型向内凹, 而最优头型 P6 则相对于初始头型向外凸; 对于水平 最大外轮廓线 C3, 最优头型 P1 和 P6 相对于初始头 型均向纵向对称面移动, 且最优头型 P1 的变化值大 于 P6。两个最优头型的鼻尖高度变化不大, 从数值 上看, P1 略有减小, P6 略有增加。 


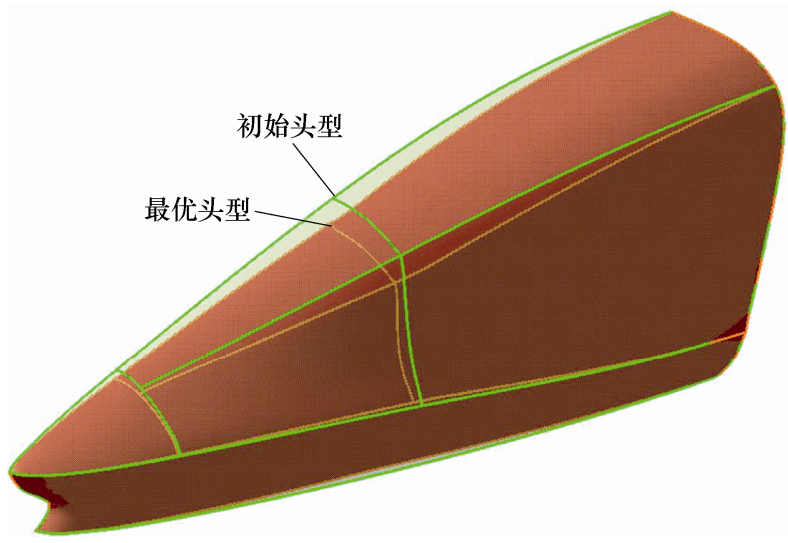

图 12 气动阻力最小的头型与初始头型的对比

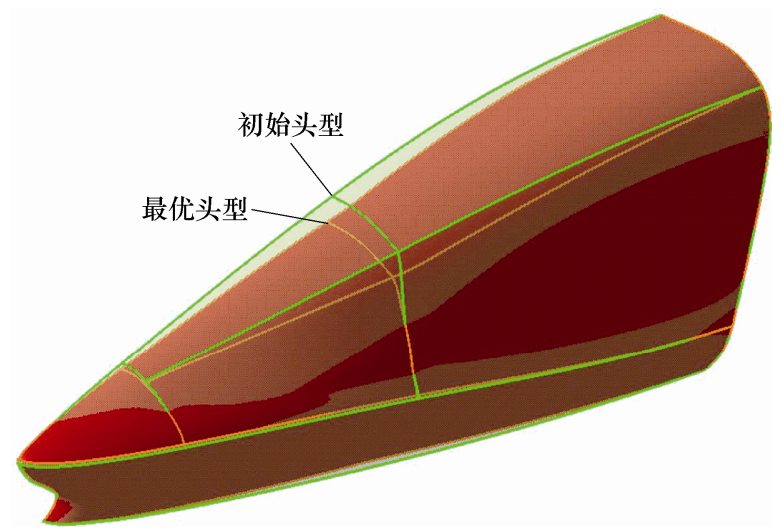

图 13 轮重减载率最小的头型与初始头型的对比

图 14 给出初始头型、气动阻力最小的头型(P1) 和轮重减载率最小的头型(P6)的流线型头型表面压力 分布云图。由图 14 可以看出, 在鼻雉区域, 初始头 型的正压区较大, 而两个最优头型的正压区则较小, 从而可以降低流线型头型区域的压差阻力, 进而降低 头车气动阻力。对于两个最优头型而言, 在流线型头 型中部, 最优头型 P6 的正压区较最优头型 P1 的正压 区大, 从而对流线型头型产生更大的下压作用, 降低 了头车气动升力, 进而降低了头车的轮重减载率。

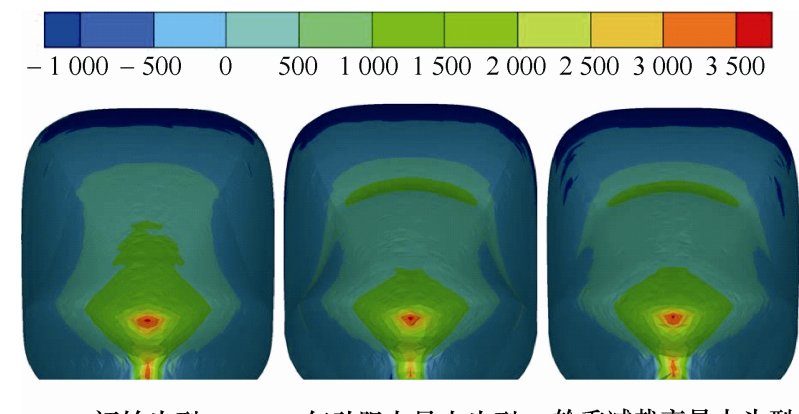

初始头型

气动阻力最小头型 轮重减载率最小头型

图 14 优化前后头型表面压力分布 $(\mathrm{Pa})$

\section{7 结论}

本文通过对高速列车流线型头型进行参数化建
模, 利用最优拉丁超立方设计和 Kriging 代理模型 建立高速列车空气动力学近似模型, 结合车辆系统 动力学模型, 以气动阻力和轮重减载率为优化目标, 采用多目标遗传算法 NSGA-II 对高速列车流线型头 型进行多目标自动寻优设计, 可以大大减少高速列 车流线型头型设计周期, 并能够获得具有更优气动 性能的高速列车流线型头型。

采用 Kriging 代理模型建立的高速列车空气动 力学近似模型具有较好的计算精度, 在 Pareto 前沿 上, Kriging 计算值与 CFD 计算值的误差在 $2 \%$ 以内, 经过多目标优化设计, 高速列车的气动阻力最多可 降低 $3.27 \%$, 轮重减载率最多可降低 $1.44 \%$, 并给 出气动阻力最小的头型及轮重减载率最小的头型, 两个最优头型的主要差异在于中部辅助控制线 C7 的变化, 前者向内凹, 而后者则略向外凸; 其次是 水平最大外轮廓线 C3 的变化, 两者均向纵向对称 面移动, 但前者移动值大于后者。

\section{参 考 文 献}

[1] 张亮, 张继业, 李田. 基于伴随方法的高速列车头型气 动优化 [J]. 机械工程学报, 2017, 53(22): 152-159.

ZHANG Liang, ZHANG Jiye, LI Tian. Aerodynamic optimization of high-speed train head based on adjoint method[J]. Jounrnal of Mechanical Engineering, 2017, 53(22): 152-159.

[2] 于梦阁, 张继业, 张卫华. 横风下高速列车流线型头型 多目标气动优化设计[J]. 机械工程学报, 2014, 50(24): 122-129.

YU Mengge, ZHANG Jiye, ZHANG Weihua. Multiobjective aerodynamic optimization design of the streamlined head of high-speed trains under crosswinds[J]. Jounrnal of Mechanical Engineering, 2014, 50(24): 122-129.

[3] DING S S, LI Q, TIAN A Q, et al. Aerodynamic design on high-speed trains[J]. Acta Mechanica Sinica, 2016, 32(2): 215-232.

[4] ZHANG J, LI J J, TIAN H Q, et al. Impact of ground and wheel boundary conditions on numerical simulation of the high-speed train aerodynamic performance[J]. Journal of Fluids and Structures, 2016, 61: 249-261.

[5] 张在中, 周丹. 不同头部外形高速列车气动性能风洞试 验研究[J]. 中南大学学报, 2013, 44(6): 2603-2608.

ZHANG Zaizhong, ZHOU Dan. Wind tunnel experiment on aerodynamic characteristic of streamline head of high speed train with different head shapes[J]. Journal of Central South Universtity, 2013，44(6): 2603-2608.

[6] 缪新乐, 李明, 姚勇, 等. 高速列车头车外形结构优化 
风洞试验研究 $[J]$. 铁道科学与工程学报, 2012, 9(2): 94-98.

MIAO Xinle, LI Ming, YAO Yong, et al. Wind tunnel test investigation on the shape optimization of head car of the high speed train[J]. Journal of Railway Science and Engineering, 2012, 9(2): 94-98.

[7] KWON H B, JANG K H, KIM Y S, et al. Nose shape optimization of high-speed train for minimization of tunnel sonic boom[J]. Jsme International Journal, 2011, 44(3): 890-899.

[8] MUÑOZ-PANIAGUA J, GARCÍA J, CRESPO A. Genetically aerodynamic optimization of the nose shape of a high-speed train entering a tunnel[J]. Journal of Wind Engineering and Industrial Aerodynamics, 2014, 130: 48-61.

[9] KRAJNOVIC S. Shape optimization of high-speed trains for improved aerodynamic performance[J]. Proceedings of the Institution of Mechanical Engineers, Part F: Journal of Rail and Rapid Transit, 2009， 223: 439-452.

[10] KU Y C, RHO J H, SU-HWAN, et al. Optimal crosssectional area distribution of a high-speed train nose to minimize the tunnel mirco-pressure wave[J]. Structural \& Multidiscciplinary Optimization, 2010， 42: 965-976.

[11] YAO S B, GUO D L, SUN Z X, et al. Parametric design and optimization of high speed train nose[J]. Optimization and Engineering, 2016, 17(3): 605-630.

[12] 熊骏, 李田, 张继业. 不同运行环境下高速列车外形气 动优化 [J]. 中国科学: 技术科学, 2016, 46(3): 313-322. XIONG Jun, LI Tian, ZHANG Jiye. Shape optimization of high-speed trains under multi running conditions[J]. Science China Technological Sciences, 2016, 46(3): 313-322.

[13] 张亮, 张继业, 李田, 等.超高速列车流线型头型多目 标优化设计 [J]. 机械工程学报, 2017, 53(2): 106-114. ZHANG Liang, ZHANG Jiye, LI Tian, et al. Multi-objective optimization design of the streamlined head shape of super high-speed trains[J]. Journal of Mechanical Engineering, 2017, 53(2): 106-114.

[14] LI Rui, XU Ping, PENG Yong, et al. Multi-objective optimization of a high-speed train head based on the FFD method[J].Journal of Wind Engineering and Industrial Aerodynamics, 2016, 152: 41-49.

[15]姚拴宝. 高速列车气动优化设计研究[D]. 北京: 中国科 学院大学, 2013.

YAO Shuanbao. Aerodynamical optimization and design of high-speed trains[D]. Beijing: University of Chinese Academy of Sciences, 2014.

[16] JIN R, CHEN W, SUDJIANTO A. An efficient algorithm for constructing optimal design of computer experiments[J]. Journal of Statistical Planning and Inference, 2005, 134(1): 268-287.

[17] 陈霞, 李否, 岳珠峰, 等. Kriging 代理模型下基于垂距 的多点取样算法 [J]. 机械工程学报, 2015，51(9): 153-158.

CHEN Xia, LI Lei, YUE Zhufeng, et al. Sampling method with multi-point sampling algorithm based on vertical distance in Kriging model[J]. Jounrnal of Mechanical Engineering, 2015, 51(9): 153-158.

[18] 于梦阁. 基于可靠性的高速列车风致安全研究[D]. 成 都：西南交通大学, 2014.

YU Mengge. Study on the wind-induced safety of the high-speed train based on the reliability[D]. Chengdu: Southwest Jiaotong University, 2014.

[19] DEB K, AGRAWAL S, PRATAP A, et al. A fast and elitist multi-objective genetic algorithm : NSGA-II [J]. IEEE Transactions Evolutionary Computation, 2002, 6(2): 182-197.

作者简介: 于梦阁, 女, 1985 年出生, 博士, 副教授。主要研究方向为 列车空气动力学及外形优化, 车辆系统动力学。

E-mail: yumengge0627@163.com

潘振宽(通信作者), 男, 1966 年出生, 博士, 教授。主要研究方向为多 体系统动力学与控制。

E-mail: zkpan@126.com 